\title{
Top properties and single top production at Tevatron
}

\author{
Meenakshi Narain* \\ Boston University, USA \\ E-mail: narain@bu.edu
}

This presentation covers the current status of measurements of top quark properties from about $350 \mathrm{pb}^{-1}$ of data collected during the Tevatron Run II by both the CDF and Dzero collaborations. We discuss the measurements of the branching fraction of the top qaurk into a $\mathrm{W}$ boson and a $b$-quark, the searches for exotic decay modes of the top quark $\left(t \rightarrow H^{+} b\right)$, the nature of the $W-t-b$ vertex. We also present the current status of the search of the single top quark signal by the Dzero experiment. This search currently sets the best 95\% C.L. upper limit on the single top quark production cross section of $5.0 \mathrm{pb}$ in the $s$-channel and $4.4 \mathrm{pb}$ in the $t$-channel.

International Europhysics Conference on High Energy Physics

July 21st - 27th 2005

Lisboa, Portugal

* Speaker. 


\section{Introduction:}

The top quark was discovered ten years ago in 1995 by the CDF and Dzero Collaborations during Run I of the Tevatron accelerator at Fermilab. During Run I, both the collaborations accumulated about $120 \mathrm{pb}^{-1}$ of data at the center of mass energy of $1.8 \mathrm{TeV}$.

The Run II of the Tevatron started in 2001, with an upgraded accelerator complex includes a new synchrotron - the main injector which replaced the old main ring and increased the capability of the Tevatron to attain higher luminosities. The c.o.m energy of the Tevatron also increased to $1.96 \mathrm{TeV}$. Both the Dzero and CDF detectors were upgraded to include various new components and electronics which resulted in enhanced capabilties for high $p_{T}$ physics. Until June 2005, the Tevatron delivered $1 \mathrm{fb}^{-1}$ of luminosity. Both Dzero and CDF detectors recorded around $800 \mathrm{pb}^{-1}$ on tape and had analyzed about $350 \mathrm{pb}^{-1}$ of luminosity. The results presented here are from the data analyzed until June 2005.

The relatively large mass of the top quark, $M_{t o p}$ (world average) $=172.7 \pm 2.9 \mathrm{GeV}$, helps probe physics at much higher energy scale than the other fermions. The top quark decays before hadronizing $\left(\tau_{\text {top }}=\sim 10^{-24} \mathrm{sec}\right.$ ) and thus we expect that its momentum and spin information is passed to its decay products. The top quark mass enters as a parameter in the calculation of the radiative corrections to other standard model (SM) observables and provides a constraint on the Higgs mass.

\section{Top Quark Decay Properties:}

2.1 Measurement of $B(t \rightarrow W b)$ :

One of the first question one may raise is: Does top quark decay $100 \%$ of the times to a W boson and a $b$-quark? Within the SM, assuming unitarity of the CKM matrix, $B(t \rightarrow W b) \sim 1$. An observation of $B(t \rightarrow W b)$ significantly different than unity would be a clear indication of new physics: non-SM top decay, non-SM background to top decay, or fourth fermion generation etc. $B(t \rightarrow W b)$ can be accessed directly in single top production. However this is not currently viable at the Tevatron as we have not yet observed single top production. In $t \bar{t}$ pair production events, we can investigate the ratio $R=\frac{B(t \rightarrow W b)}{B(t \rightarrow W q)}$ which is related to $V_{t b}^{2}$. In the standard model, $V_{t b}^{2}=0.998$.

$R$ can be measured by comparing the number of $t \bar{t}$ candidates with 0,1 and 2 jets tagged. In the 0 -tag bin, a discriminant variable exploiting the differences in event kinematics between $t \bar{t}$ and background is used to increase the signal significance. Using $160 \mathrm{pb}^{-1}$ of data, CDF determines $R=1.12+0.27-0.13$ or $R>0.61$ at $95 \%$ C.L.. Dzero analyzes $230 \mathrm{pb}^{-1}$ and measures $R=$ $1.03+0.19-0.17$ or $R>0.64$ at $95 \%$ C.L..

The statement that $R$ is measured to be close to 1 , only implies that the top quark decays to a bquark $t \rightarrow X+b$. Is $X$ a W Boson or could $X$ be the charged Higgs as predicted by generic two Higgs Doublet Models? CDF has carried out a search for Charged Higgs in top quark decays $(t \rightarrow H+b)$. If the charged Higgs mass is less than $m_{t}-m_{b}$ then, $t \rightarrow H+b$ competes with $t \rightarrow W+b$ and results in $\mathrm{B}(t \rightarrow W b)$ less than 1 . Charged Higgs decays are different than $\mathrm{W}$ decays and affect the $t \bar{t}$ production cross section measurements in different channels (dileptons, lepton+jets, lepton+tau). A simultaneous fit is performed to the observation in all channels to determine a model-dependent exclusion region in $\left(\tan \beta, M_{H}^{ \pm}\right)$. 


\subsection{Properties of the $\mathrm{W}$-t-b vertex:}

The massiveness of the top quark raises the question: are there new interactions at energy scales near Electroweak Symmetry Breaking? A measurement of the helicity of the $\mathrm{W}$ boson gives a way to examine the nature of the t-b-W vertex and provide a stringent test of standard model. A general form of the Lagrangian for the $t-b-W$ interaction is given in ref [1]. It contains four form factors; $f_{1}^{L}, f_{1}^{R}$ that parameterize the $\mathrm{V}$-A and $\mathrm{V}+\mathrm{A}$ interactions, and $f_{2}^{L}, f_{2}^{R}$ which reflect the strength of and anomalous weak magnetic moment. In the SM, the coupling of $\mathrm{W}$ to fermions is purely V-A, and therefore the only non zero form factor is $f_{1}^{L}$. If the t-b-W couplings are standard, then top quark decays to left handed $\left(W_{-}\right)$or to longitudinal $\left(W_{0}\right) \mathrm{W}$ bosons. In the presence of non-standard coupling such as $\mathrm{V}+\mathrm{A}$, some admixture of right handed $\mathrm{W}$ bosons $\left(W_{+}\right)$is expected.

The polarization of the $\mathrm{W}$ bosons can be analyzed via the angular distributions of its decay products. If we define $\theta^{*}$ as the angle of the decay positron in the $\mathrm{W}$ rest frame, with the polarization axis defined by the direction of the $\mathrm{W}$ boson in the top rest frame, then the angular distribution of the lepton with respect to the polarization of the $W$ is defined by

$$
w\left(\cos \theta^{*}\right)=F_{-} \frac{3}{8}\left(1-\cos \theta^{*}\right)^{2}+F_{0} \frac{3}{8}\left(1-\cos ^{2} \theta^{*}\right)+F_{+} \frac{3}{8}\left(1+\cos \theta^{*}\right)^{2}
$$

where $F_{-}, F_{0}, F_{+}$are the fractions of left handed, longitudinal, and right handed $W$ bosons. In the $\mathrm{SM} F_{-}, F_{0}, F_{+}$are expected to be $0.7,0.3,0$ respectively.

The $p_{T}$ spectrum of the lepton also carries information about the helicity of the $\mathrm{W}$ boson. Leptons emitted from the longitudinal $\mathrm{W}$ bosons are perpendicular to the $\mathrm{W}$ direction and hence have harder $p_{T}$ compared to those emitted from the left-handed $\mathrm{W}$ bosons. The leptons from lefthanded $\mathrm{W}$ bosons are emitted opposite to $\mathrm{W}$ boson direction, thus leading to softer $p_{T}$ spectra.

CDF and Dzero have analyzed both the $p_{T}$ spectrum of the leptons and the angular distribution using di-leptons and lepton+jet events respectively. A likelihood analysis of the lepton $p_{T}$ spectrum leads to the result $F_{+}=0.13 \pm 0.20$ (stat) \pm 0.16 (syst); $F_{+}<0.28$ at $95 \%$ C.L. (Dzero) and $F_{0}=0.27+0.35-0.21($ stat + syst $) ; F_{0}<0.88$ at $95 \%$ C.L. $(\mathrm{CDF})$. From the analysis of the angular distribution of the leptons, Dzero obtains: $F_{+}=0.04 \pm 0.11$ (stat) $\pm 0.06($ syst $) ; F_{+}<$ 0.25 at $95 \%$ C.L.. The measurements are consistent with the SM prediction: $F_{0}=0.7, F_{+}=0$.

\section{Single Top Quark Searches at Dzero:}

Single top quarks are produced through a Wtb vertex involving (V-A) electroweak coupling. In contrast, $t \bar{t}$ pairs are produced via strong interactions. The two main production processes for single top quark at the Tevatron are s-channel $(p \bar{p} \rightarrow t b+X)$ and t-channel $(p \bar{p} \rightarrow t q b+X)$. The production cross sections have been calculated[2] to be $0.88 \pm 0.14 \mathrm{pb}$ for the s-channel and $1.98 \pm 0.30$ $\mathrm{pb}$ for the t-channel (for $m_{t}=175 \mathrm{GeV}$ ). We have not yet observed single top quark production. This search is much more challenging than $t \bar{t}$ production due to smaller cross sections and larger backgrounds.

One of the primary interests in establishing the cross section for single top production is that it is directly sensitive to the transition width of $\mathrm{t}$ to $\mathrm{Wb}$, and consequently to $V_{t b}$. There are also interesting issues pertaining to the existence of the right-handed couplings and polarization. The sand $\mathrm{t}$-channels are sensitive to different new physics and hence can be used to distinguish between 
various exotic models (FCNC, Top Flavor, 5th generation etc). They are also the main irreducible background to associated Higgs production.

Search for single top quark starts with selection of events with a b quark and a $\mathrm{W}$ boson, which subsequently decays leptonically $(W \rightarrow e / \mu v)$ from a $230 p b^{-1}$ dataset. The signal signature consists of an isolated high transverse momentum electron or muon, significant missing transverse energy due to the neutrino and two or three high transverse momentum jets, of which at least one is identified as a $b$-quark jet. The largest backgrounds are from the associated production of a $\mathrm{W}$ boson with jets $(\mathrm{W}+\mathrm{jets})$ and top pair production. Additional multijet background comes from events containing a mis-identified isolated lepton associated with hadronic jets.

In order to reduce the backgrounds, both kinematical and topological information in the event was used. Some of the many variables which were tried for discriminating against the backgrounds were: Jet $p_{T}$ for different jets; b-tag information of the jet; $\mathrm{H}$ (total energy); $H_{T}$ (transverse energy); M (invariant mass); $M_{T}$ (transverse mass); Summing over various objects in the event; Jet-jet separation; Jet pseudorapidity (t-channel); Top quark spin etc.

Four different analysis techniques were employed to separate the signal from the background: Cut based, Neural Networks (NN), Decision Trees(DT) and Likelihood (LD) Discriminant. The first three use the same structure and optimize separately for s-channel and t-channel signal. They focus on rejecting dominant backgrounds: $\mathrm{W}+\mathrm{jets}$ and $t \bar{t}$ by training the neural networks or decision trees separately for each background, based on same set of discriminating variables. Finally, there are eight separate sets of cuts or networks or trees. The likelihood discriminant method utlizes a larger sample of data $\left(370 \mathrm{pb}^{-1}\right)$ and uses a similar set of variables to construct discriminants for the signal and backgrounds.

Since the observed data are consistent with the background predictions for all channels and techniques used, we therefore proceed to set upper limits on the single top quark production cross section shown in Table 1. These upper limits represent significant improvements over previously published results[3], due to larger data set analyzed, and the use of multivariate analysis techniques. It is interesting to note that they approach the region of sensitivity for models for new physics such as a fourth quark generation scenario or flavor-changing neutral-currents.

\begin{tabular}{c|c|c|c|c|c|c|c|c}
\hline \hline & Theory & \multicolumn{2}{|c|}{ Run I } & \multicolumn{5}{|c}{ Run II } \\
\hline & & CDF & Dzero & CDF & \multicolumn{4}{|c}{ Dzero } \\
\hline & & & & & Cut & DT & NN & LD \\
s-channel & $0.88 \mathrm{pb}( \pm 8 \%)$ & $<18 \mathrm{pb}$ & $<17 \mathrm{pb}$ & $<14 \mathrm{pb}$ & $<10.6 \mathrm{pb}$ & $<8.3 \mathrm{pb}$ & $<6.4 \mathrm{pb}$ & $<5.0 \mathrm{pb}$ \\
$\mathrm{t}-$-channel & $1.98 \mathrm{pb}( \pm 11 \%)$ & $<13 \mathrm{pb}$ & $<22 \mathrm{pb}$ & $<10 \mathrm{pb}$ & $<11.3 \mathrm{pb}$ & $<8.1 \mathrm{pb}$ & $<5.0 \mathrm{pb}$ & $<4.4 \mathrm{pb}$ \\
\hline \hline
\end{tabular}

Table 1: 95\% C.L. upper limit on single top production cross section.

\section{References}

[1] G. Kane, C.-P. Yuan, and G. Ladinsky, Phys. Rev. D 45, 124, (1992).

[2] B. W. Harris et al., Phys. Rev. D 66, 054024 (2002); Z. Sullivan, Phys. Rev. D 70, 114012 (2004).

[3] Dzero Collaboration, B. Abbott et al., Phys. Rev. D 63, 031101 (2001); CDF Collaboration, D. Acosta et al., Phys. Rev. D 71, 012005 (2005). 